\title{
Electroencephalography in Psychiatric Surgery: Past Use and Future Directions
}

\author{
Karim Mithani ${ }^{a, b}$ Ying Meng ${ }^{a}$ Agessandro Abrahao $^{a}$ Mirriam Mikhail $^{b}$ \\ Clement Hamani ${ }^{a}$ Peter Giacobbe ${ }^{a}$ Nir Lipsman ${ }^{a}$ \\ a Sunnybrook Research Institute, Toronto, ON, Canada; ${ }^{\text {b}}$ Faculty of Medicine, University of Toronto, \\ Toronto, ON, Canada
}

\section{Keywords}

Electroencephalography · Psychiatric surgery · Biomarker

\begin{abstract}
The last two decades have seen a re-emergence of surgery for intractable psychiatric disease, in large part due to increased use of deep brain stimulation. The development of more precise, image-guided, less invasive interventions has improved the safety of these procedures, even though the relative merits of modulation at various targets remain under investigation. With an increase in the number and type of interventions for modulating mood/anxiety circuits, the need for biomarkers to guide surgeries and predict treatment response is as critical as ever. Electroencephalography (EEG) has a long history in clinical neurology, cognitive neuroscience, and functional neurosurgery, but has limited prior usage in psychiatric surgery. MEDLINE, Embase, and PsycINFO searches on the use of EEG in guiding psychiatric surgery yielded 611 articles, which were screened for relevance and quality. We synthesized three important themes. First, considerable evidence supports EEG as a biomarker for response to various surgical and non-surgical therapies, but large-scale investigations are lacking. Second, intraoperative EEG is likely more valuable than surface EEG for guiding
\end{abstract}

target selection, but comes at the cost of greater invasiveness. Finally, EEG may be a promising tool for objective functional feedback in developing "closed-loop" psychosurgeries, but more systematic investigations are required.

(c) 2019 S. Karger AG, Basel

\section{Introduction}

Psychiatric surgery involves neurosurgical treatment of mental illnesses that are debilitating and not treatable by conventional interventions, such as medications, therapy, or non-invasive neuromodulation [1]. The field has a controversial past, plagued by imprecise techniques, lax selection criteria, insufficient ethical oversight, and significant morbidity and mortality. However, technological advancements over the past several decades, along with a better understanding of the neurobiology of mental illness and a persistent need for interventions for refractory cases, have spurred a re-emergence of the field [2]. Image-guided, minimally invasive interventions such as gamma knife radiosurgery and focused ultrasound, as well as reversible neuromodulation procedures such as deep brain stimulation (DBS), offer promising treatment options for many patients for whom conventional therapies have failed [3].

\section{KARGER}

(c) 2019 S. Karger AG, Basel

E-Mail karger@karger.com

www.karger.com/sfn
Nir Lipsman, MD, PhD

Hurvitz Brain Sciences Research Program, Sunnybrook Research Institute 2075 Bayview Ave., Room A1-39

Toronto, ON M4N 3M5 (Canada)

E-Mail nir.lipsman@utoronto.ca 
Despite advancements in structural and functional neuroimaging that can help guide these procedures, there is an urgent need for real-time, physiological feedback to improve precision and accuracy for surgical targeting. This holds true for all psychosurgery techniques. For example, although focused ultrasound capsulotomy can be performed under MRI guidance, allowing for immediate visualization of the lesion, there is currently no intraprocedural functional feedback $[3,4]$. Therefore, even if the formation of a lesion is visually confirmed, its effect on the patient's neuropsychiatric functioning - the primary outcome of interest - is not immediately clear. Electroencephalography (EEG) may serve as a useful biomarker for monitoring neuropsychological changes during the procedure and to predict treatment response. Furthermore, neurophysiological response to sublesional temperature sonications would help tremendously in improving target selection. This article comprehensively reviews the medical literature on the use of EEG in psychiatric surgery, discusses its applications as a biomarker of treatment response, and comments on future possibilities of the technology.

\section{Methods}

Our search was designed to capture as many relevant articles as possible, prioritizing sensitivity over precision. Subject headings and text words related to our topic were manually curated. Relevant synonyms were identified using PubMed's Automatic Term Mapping feature [5], searching the terms in encyclopaedias for synonyms, and perusing Medical Subject Headings (MeSH) scope notes. Furthermore, a test set of 4 articles was developed and agreed upon by all authors, based on their known relevance to our topic (online suppl. material S1; for all online suppl. material, see www.karger.com/doi/10.1159/000500994). Subject headings for each of these articles were identified using the Yale MeSH Analyzer [6] and incorporated into the search strategy. Equivalent EMTREE and PsycINFO Thesaurus subject headings were identified wherever possible.

Once all terms and concepts were identified, they were combined with Boolean operators; synonyms were combined with "OR," while distinct search concepts were linked with "AND" (Fig. 1). After briefly reviewing some of the results from our first search, the search phrase was modified to include more subject headings that were initially missed.

Searches were performed iteratively on MEDLINE, Embase, and PsycINFO, using the Ovid ${ }^{\circledR}$ platform, with the last search conducted on July 17,2018 . Slightly different search phrases were used in each database because of differences in subject headings and organization. All 4 of the articles in the test set appeared in the final search results, reaffirming the sensitivity of our approach. A detailed search strategy can be found in online supplementary material S2.

All articles underwent an initial title and abstract screen for relevance, followed by a full-text review. Results from included studies were extracted and textually summarized. For especially

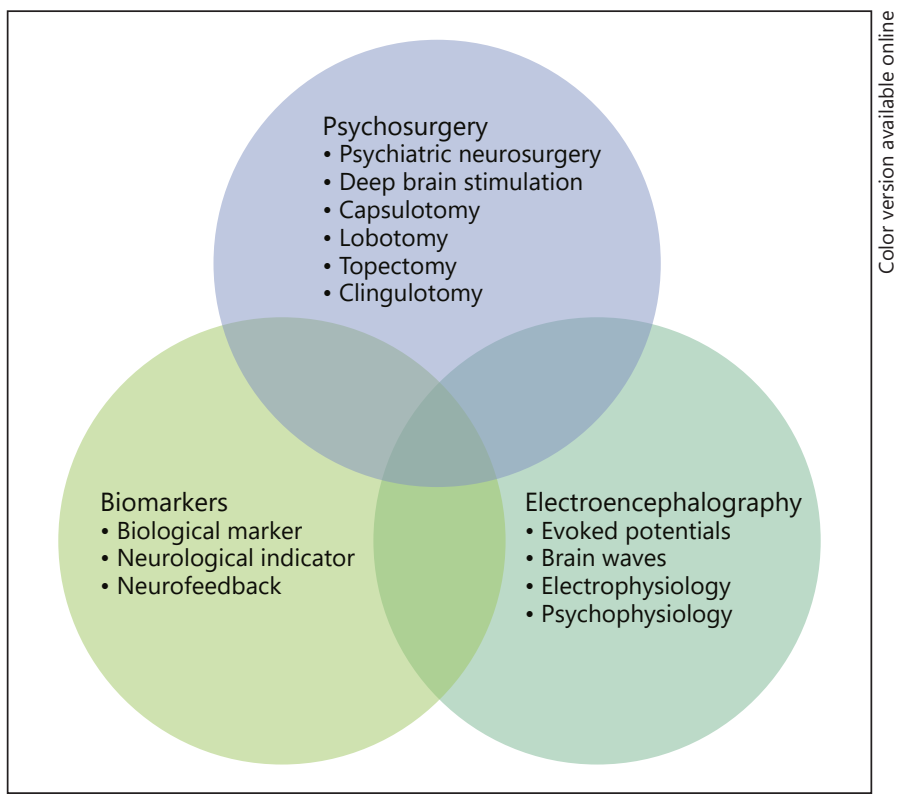

Fig. 1. Graphical overview of our search strategy; titles represent distinct search concepts and bullets represent select synonyms.

relevant and/or high-quality articles, a citation pearl growing strategy was used to expand the list of relevant literature.

In the interest of comprehensiveness, numerous sources of grey literature were also pursued. We searched clinical trial records (ClinicalTrials.gov, WHO International Clinical Trials Registry Platform, and the Cochrane Central Register of Controlled Trials), conference proceedings (Scopus "Conference Papers" documents, as well as proceedings from the American Society for Stereotactic and Functional Neurosurgery (ASSFN) and World Society for Stereotactic and Functional Neurosurgery (WSSFN) conferences), theses and dissertations (ProQuest Dissertations \& Theses Global), government documents (Publications Canada), corporate reports, newspapers and magazines (ProQuest), and practice guidelines from the ASSFN and WSSFN for relevant documents.

We excluded any studies that focused primarily on non-humans, as the goal of this review was to explore EEG correlates of psychiatric surgery in humans only. In addition, we removed any articles which were not written in English and for which an English translation was not available. Finally, no review articles were included in this narrative synthesis; however, they were occasionally used for citation pearl growing to identify important studies in the field.

Mendeley software (version 1.19; Elsevier, The Netherlands) was used for deduplication and reference managing. This search strategy was designed in consultation with a health sciences librarian.

\section{Results}

In total, our search returned 611 articles, 199 of which were duplicates. Amongst the 412 unique articles, 56 were not available in English and 21 primarily involved non- 
Fig. 2. Overview of article identification and selection; adapted from Preferred Reporting Items for Systematic Reviews and Meta-Analyses (PRISMA) guidelines [77].

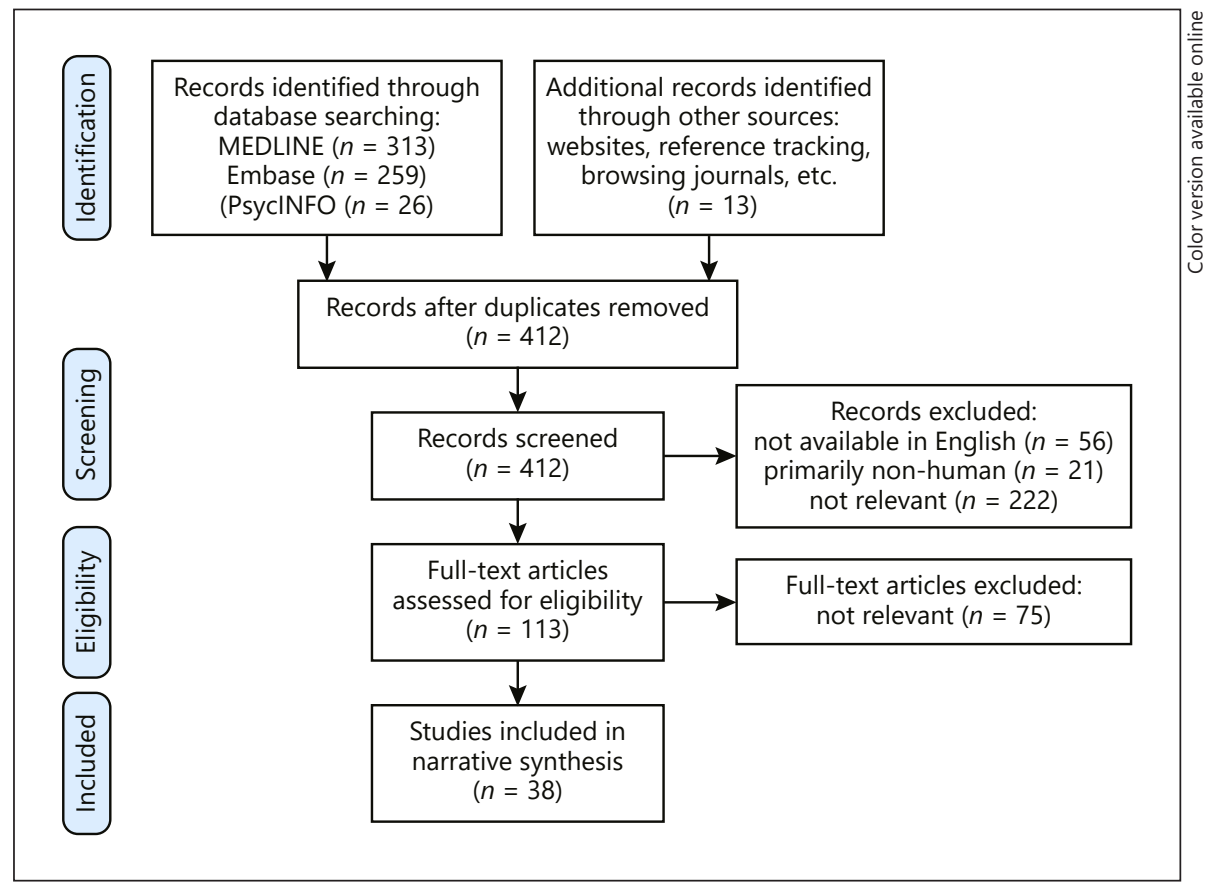

humans and were therefore excluded. The remaining 335 articles were screened for relevance based on their titles and abstracts, and 113 proceeded to full-text review. Ultimately, 38 articles were included in this narrative synthesis (Fig. 2).

Grey literature searches yielded a total of 268 items, all of which were screened for inclusion (online suppl. material S3). Ultimately, 4 grey literature items were included in the narrative synthesis.

\section{EEG in Psychiatric Surgery}

The use of EEG in psychiatric surgery is almost as old as the field itself, with studies dating back to the early 1940s (Hutton et al. [7], 1941; Davis [8], 1941; Cohn [9], 1945; Levin et al. [10], 1949; Grevilie and Last [11], 1947; Lennox and Coolidge [12], 1949; Stevens and Mosovich [13], 1947). Jean Talairach, possibly the first person to perform anterior capsulotomy for a psychiatric indication, endorsed the use of intraoperative EEG and electrocorticography in his procedures. He recommended using EEG to not only better understand psychiatric illness, but also to identify targets for stereotactic lesioning, termed "stereo-encephalography" $[14,15]$. Early studies consistently report distinct changes in EEG patterns immediately after prefrontal lobotomy that were in some reports associated with treatment response (Table 1). These changes were most pronounced in the frontal lobes, and frequently involved heightened slow wave activity. Notably, the vast majority of post-operative EEG changes were temporary and returned to normal within weeks to months. Cohn [9] (1945) attributed the immediate increase in slow delta and theta waves after frontal undercutting to operation-induced brain damage, as it correlated with the "akinetic" state seen in most patients after the procedure. In support of this, EEG changes returned to baseline with resolution of the akinetic state. Others posited that these changes were a result of disruption of abnormal cortico-thalamo-cortical impulses, resulting in reduced prefrontal activity $[12,13]$.

Despite generally similar findings, there were some distinct differences in post-operative EEG changes noted amongst these early psychiatric surgery reports. This variability is at least in part due to differences in surgical procedures and EEG recording techniques, but may also reflect differences in ablation targets. For example, in 1950 Henry [16] described EEG patterns after transorbital lobotomy that were very different from those noted for other prefrontal lobotomy procedures. In particular, he reported a distinct lack of "true slow wave activity" after transorbital lobotomy, and in fact found a somewhat irregular but mostly dominant alpha wave pattern. 
Table 1. Summary of documented EEG changes as a result of psychosurgery

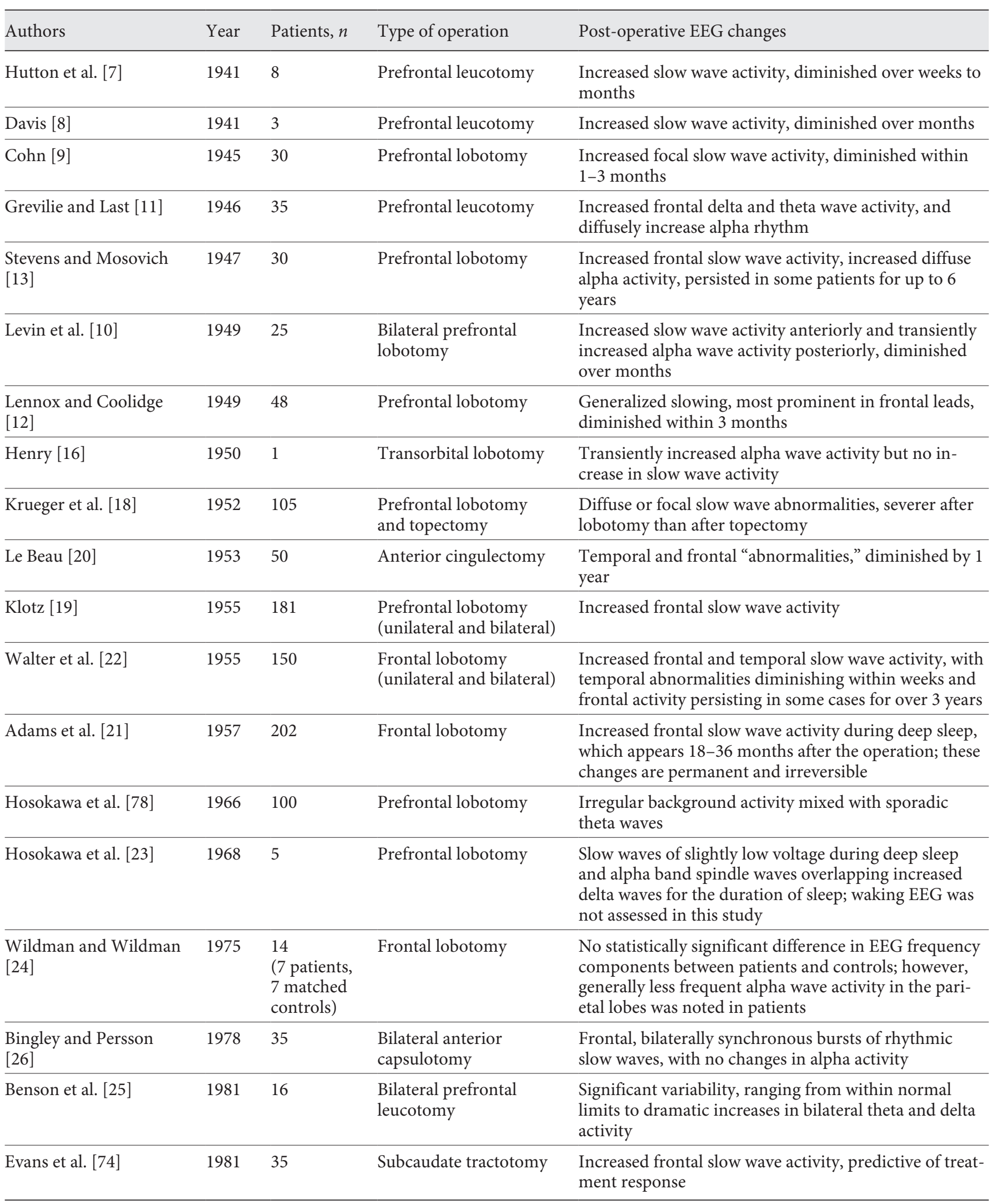


The 1946 Columbia-Greystone project also found EEG abnormalities in most patients after topectomy for psychiatric disorders. Again, these changes were predominantly transient, and disappeared after approximately 4 months [17]. Krueger et al. [18] (1952) confirmed these findings in their trial comparing prefrontal lobotomy and topectomy for the treatment of chronic, advanced schizophrenia. EEG recordings prior to the procedure were relatively normal, but immediately afterwards showed slow wave abnormalities - either diffuse or focal - characteristic of cortical damage. In their study, patients with lobotomy had more EEG slow wave abnormalities than the topectomy group, but also had considerably better response to treatment. EEG changes were not systematically associated with the level of clinical improvement, and abnormalities typically resolved spontaneously within several months. Subsequent studies on prefrontal lobotomy and anterior cingulectomy found similar patterns of EEG changes [19], albeit the latter with more temporal lobe involvement rather than just frontal lobe [20].

In contrast to transient changes, persistent EEG changes have also been observed. A study of bilateral or unilateral lobotomy for 27 patients with various psychiatric disorders found persistently elevated frontal slow wave activities 3 years after surgery (Walter et al. [21], 1955). Another long-term study of 202 bilateral prefrontal lobotomy patients lent further support to the presence of persistent post-surgical EEG abnormalities. Specifically, Adams et al. [22] (1957) found that the most consistent and marked EEG change after prefrontal lobotomy was asynchronous interhemispheric electrical activity of the frontal lobes during deep sleep. While waking post-operative changes in their patients were mild and quick to resolve, these distinct changes in sleep did not even appear until at least 18 months after the operation. Notably, this change manifested in all cases by 36 months post-operatively, and was thought to be permanent and irreversible. The mechanism behind the abnormal recording and the temporal delay may involve delayed degeneration of commissural fibres linking the left and right nuclei after the operation. Other studies have since confirmed sleepspecific EEG disturbances after prefrontal lobotomy [23]. Very long-term follow-ups, in the order of decades after the original procedure, have generally failed to find persistent waking changes, albeit with some exceptions $[24,25]$.

A study of stereotactic bilateral anterior capsulotomy for obsessive compulsive disorder (OCD) also found distinct abnormalities after surgery from what appeared to

EEG in Psychiatric Surgery: Past Use and Future Directions be generally normal at baseline [26]. To date, this is the only study to assess EEG in bilateral anterior capsulotomy for OCD. Specifically, in keeping with much of the aforementioned literature, rhythmic, slow wave activity of $<5 \mathrm{~s}$ in duration and $\sim 100-300 \mu \mathrm{V}$ in amplitude was noted 10 days after the procedure. These abnormalities were bilaterally synchronous, independent of lesion size, and most prominent frontally, but also observed temporally and centrally. Upon re-examination 1-2 years after the surgery, most patients had returned to normal, although a few had persistent, slow wave frontal activity. To date, this is the only study to assess EEG in bilateral anterior capsulotomy for OCD.

In summary, resting-state EEG studies after psychiatric surgery have generally shown postoperative abnormalities, including increased slow delta and theta wave activity. Unfortunately, technological limitations at the time, as well as a lack of appropriate controls and blinding, make it difficult to discern whether post-operative EEG patterns were truly due to functional changes as a result of the surgery or simply a reflection of neuronal damage caused by surgical manipulation. The former would constitute valuable neurophysiological feedback to guide psychiatric neurosurgery, whereas the latter would provide little-to-no useful information.

\section{Event-Related Potentials in Psychiatric Surgery}

Event-related potentials (ERPs), as opposed to restingstate EEG activity, have a better signal-to-noise ratio and are generally less affected by diffuse brain damage [27, 28]. Accordingly, ERPs may provide a more accurate overview of electrophysiological correlates of functional changes after psychiatric surgery, rather than non-specific changes due to surgical manipulation alone. Several studies have attempted to delineate the changes in ERPs as a result of psychiatric surgery. Amongst the first ERPs described was the contingent negative variation (CNV): a slow electrical potential elicited by a "warning" stimulus, which prepares the subject for an "imperative" stimulus requiring some sort of motor or decision response (Walter et al. [29], 1964; Kappenman and Luck [30], 2011). Early studies reported conflicting results, with some noting no $\mathrm{CNV}$ changes with interruption of thalamocortical fibres [31], while others found a distinct absence of specific CNV patterns in certain psychiatric surgery patients [32] and even associated it with treatment response [33].

A different type of ERP, known as error-related negativity (ERN), has been used to assess anterior mid-cingulate cortex activity in severe alcohol addiction [34]. The 
anterior mid-cingulate cortex forms part of what the authors describe as an internal "performance monitoring system" that imparts the ability to monitor action outcomes and derive appropriate behavioural adaptations in response to alcohol cravings or related cues. The ERN is associated with errors of commission and is thought to reflect the activity of the anterior mid-cingulate cortex. The investigators believed that this structure and its associated functional network were hypoactive in certain patients with acute alcohol addiction. Accordingly, they performed DBS of the nucleus accumbens (NAc), which is part of the performance monitoring system, and used the ERN, an indicator of anterior mid-cingulate cortex activity, as a non-invasive marker of treatment response. The authors demonstrated a consistent and sustained, statistically significant increase in ERN with DBS of the NAc, which was associated with clinical improvement [34]. Subsequent studies on DBS for alcohol addiction have used intracranial and surface EEG, recorded after implantation but prior to stimulation, to identify local field potentials that further clarify the role of the NAc and its functional networks in mediating the disorder $[34,35]$.

The ERN has also been studied in patients with Huntington's disease undergoing DBS of the globus pallidus, which is thought to interact with the anterior cingulate cortex (ACC) in driving behavioural adaptation after committing errors [36]. Baseline ERN is typically reduced in this population, but DBS appeared to normalize it to the point that its amplitude was no different from that of controls. Furthermore, the ERN disappeared when stimulation was turned OFF. This finding suggests that DBS of the globus pallidus may improve not only motor symptoms but also certain cognitive functions in patients with Huntington's disease.

Evidently, ERPs may provide accurate measures of neuropsychological changes as a result of psychiatric surgery. Although the neurobehavioural correlates of ERPs are a prominent area of neuroscientific research, their utility in guiding and prognosticating psychiatric surgery have not been systematically investigated.

\section{Intraoperative EEG}

Intraoperative EEG has a rich history in psychiatric neurosurgery. Early studies used intracerebral electrography to narrow down the target of prefrontal leucotomy in an attempt to reduce adverse events [37]. More recently, the emergence of DBS as a treatment for refractory mental illnesses has provided a compelling opportunity for deep electrophysiological recordings.
Briefly, in DBS, electrodes are implanted within specific structures or tracts in order to neuromodulate abnormal over- or under-activity [38]. The electrical activity of these structures can be directly measured through these electrodes, which provides a salient opportunity for elucidating disease pathophysiology and augmenting surgical targeting. For example, in treating a case of refractory major depressive disorder (MDD), Jiménez et al. [39] (2005) systematically varied stimulation parameters of an electrode in the inferior thalamic peduncle while monitoring evidence of cortical recruitment and regional direct current shifts on EEG. They observed that stimulation of the inferior thalamic peduncle and centromedian nucleus at certain parameters resulted in similar cortical synchronization and direct current shifts in the bilateral frontopolar leads. This was justified by citing that both structures are "anatomically and physiologically related to nonspecific thalamic and thalamo-orbitofrontal systems." Stimulating the ventromedial nucleus of the hypothalamus and fornix also resulted in similar EEG changes, but were more generalized, involving frontal, central, and temporal leads. Intraoperative intracranial and surface EEG recordings, in conjunction with pictures of drugs, have also been used to augment targeting for DBS in a patient with heroin addiction [40].

In addition to guiding surgeries, intraoperative EEG and electrocorticography have enabled a better understanding of circuit disorders underlying mental illnesses. These approaches help identify new surgical or neuromodulation targets. For example, neuronal activity recorded during DBS for OCD elucidated a key role played by aberrant caudate nucleus activity in the pathophysiology of the disease [41]. In addition, intracranial and surface EEG recordings in DBS patients with MDD have helped to elucidate the role of the NAc in negative reinforcement-guided adjustments in behaviour [42]. Intraoperative electrocorticography has also been used to study the role of low-frequency oscillations and reduced alpha activity in the posterior hypothalamus in mediating pathologic aggression [43]. One study in OCD patients undergoing DBS has suggested that dysfunctional neuronal synchronization dynamics in frontostriatal circuits, as indicated by aberrant local field potentials in frontal electrodes, is significantly more prominent when stimulation is turned OFF rather than ON. They posit that interventions that disrupt this pathological connectivity, such as DBS, may be the most effective method in remediating treatment-resistant symptoms [44].

To date, nearly all investigations of intraoperative EEG in psychiatric surgery have focused on DBS and invasive 
forms of neurophysiology. Newer, incisionless approaches such as gamma knife radiosurgery and focused ultrasound do not allow for intracranial EEG recordings, but may still benefit from intraoperative electrophysiological guidance. Surface EEG during these procedures may provide valuable functional feedback to guide surgeries, similar to that of intracranial EEG in DBS operations, and its feasibility and utility should therefore be investigated.

\section{EEG as a Biomarker of Treatment Response}

In addition to its utility in diagnostics and surgical guidance, EEG is promising as a biomarker of disease severity and predictor of treatment response. Its widespread availability in hospitals and clinics, portability, and relatively low cost make it an ideal tool for this purpose. EEG has been used to study a number of psychiatric conditions, and an overview of the literature on MDD is presented here as an illustrative example.

\section{Major Depressive Disorder}

MDD is the most common mood disorder, and is typically characterized by persistent low mood, hopelessness, guilt, and reduced energy. Despite multiple rounds of antidepressant therapy, up to $30-40 \%$ of patients remain symptomatic [45]. MDD is a highly heterogeneous disease, with many subtypes and varying levels of severity, which can complicate diagnosis and treatment plans. EEGs of MDD patients have been extensively studied and reviewed elsewhere [46-55].

Meta-analysis of numerous, multimodal studies suggests that response to antidepressants, electroconvulsive therapy, repetitive transcranial magnetic stimulation, or sleep deprivation therapy is predicted by increased ACC activity at baseline $[46,48]$. EEG can potentially be used to detect this elevated ACC activity, for example by identifying increased theta activity at electrode sites Fz and/or $\mathrm{FCz}$, or more precisely using a technique known as low resolution electromagnetic tomography (LORETA) [56]. This involves computing current distribution throughout the brain and, under a set of physiological assumptions about neuronal activity, generating 3-D tomography with relatively low spatial resolution. The advantage of LORETA is that it combines the high temporal resolution of EEG with crude spatial localization of the electrophysiological signal. It has repeatedly demonstrated robust identification of generators of EEG frequency components and ERPs, in both healthy and diseased states [57]. However, LORETA has also been criticized for specific inaccuracies and for the large number of neuroanatomical and electrophysiological constraints that it employs. Nevertheless, LORETA remains amongst the most effective methods of localizing EEG signals to specific areas of the brain [57].

Some other biomarkers of antidepressant response include: increased alpha power, right frontal dominant alpha asymmetry, decreased theta band activity, responses to P300 and N100 ERPs, theta concordance - a complex combination of absolute and relative theta power in QEEG - and the antidepressant treatment response index, which combines relative and absolute frontal alpha and theta power at different time points $[47,49,51,52$, 54]. Potential EEG biomarkers of poor treatment response include: increased slow (i.e., theta or delta) power, decreased frontal, parietal, and occipital alpha power, left frontal dominant alpha asymmetry, and slow individual alpha peak frequency $[46,49,51]$. More recently, modern machine learning approaches employing large datasets have identified coherence between frontotemporal electrodes in the alpha and beta frequencies as particularly strong predictors of antidepressant treatment response $[58,59]$.

Certain EEG biomarkers have also been shown to predict positive treatment response in neuromodulation studies of MDD, which target the same neuronal circuits as some lesional procedures (Table 2). These biomarkers include: low theta power in the subgenual ACC, high individual alpha peak function, prefrontal theta/delta concordance, and reduced parieto-temporal alpha power, although some of these have failed to replicate in other studies $[50,52]$.

\section{Deep Brain Stimulation}

As previously mentioned, the ability to precisely record electrical activity using intracranially implanted electrodes, as well as surface EEG with varying stimulation parameters, provides a unique opportunity to explore EEG as a biomarker of DBS treatment response. Data from MDD patients who underwent DBS of the subgenual cingulate gyrus identified several potential EEG biomarkers of surgical outcomes. The hemispheric power and synchronization asymmetry was significantly different in responders and non-responders, when the stimulation was turned both ON and OFF, 3-6 years after implantation [60]. Frontal theta power asymmetry negatively correlated with treatment-related change in depression severity, while parietal alpha power asymmetry showed the opposite relationship. Similarly, mean frontal synchronization asymmetry positively correlated 
Table 2. Summary of EEG biomarkers of response to antidepressant therapy

\begin{tabular}{ll}
\hline Good response & Poor response \\
\hline $\begin{array}{l}\text { Pharmacotherapy } \\
\text { Elevated frontal midline theta activity }\end{array}$ & Increased theta or delta power \\
Increased alpha power & Decreased frontal alpha power \\
Right frontal dominant alpha asymmetry & Decreased parietal alpha power \\
Decreased theta band activity & Decreased occipital alpha power \\
Responses to P300 and N100 ERPs & Left frontal dominant alpha asymmetry \\
Antidepressant treatment response index & Slow individual alpha peak frequency \\
\hline
\end{tabular}

Neuromodulation

Low theta power in the subgenual ACC

High individual alpha peak function

Increased prefrontal theta/delta concordance

Reduced parieto-temporal alpha power

with the change in depression severity before and after DBS, while mean parietal synchronization asymmetry exhibited the opposite association. Furthermore, a set of healthy controls without MDD or any DBS implants showed a hemispheric asymmetry pattern similar to treatment responders, but opposite to that of non-responders. Interestingly, the asymmetry pattern seen in non-responders - increased left-relative-to-right parietal alpha activity and decreased left-relative-to-right frontal theta activity - is typical of depressed individuals [60]. These findings not only further delineate the mechanism of action of DBS in treating refractory MDD, but also provide a potentially useful biomarker to non-invasively assess treatment response. Other EEG correlates of neuromodulatory treatment of MDD have been reviewed elsewhere [61, 62].

Local field potentials from the bed nucleus of the stria terminalis in refractory OCD and MDD patients, recorded from still-externalized DBS electrodes, can distinguish treatment-resistant MDD from OCD [63]. Furthermore, mean alpha activity in the subgenual ACC and bed nucleus of the stria terminalis are positively correlated with self-reported symptom severity in MDD, but not in OCD. In addition, mean beta power in the subgenual ACC has shown inverse correlations with depression severity [64]. Another study on DBS of the subgenual ACC for treatment-resistant depression showed enhanced beta desynchronization as a result of increased empathic responses to negative stimuli at baseline [65]. Furthermore, suppression of beta band oscillatory activity was correlated with symptom severity. Interestingly, 6 months of DBS of the subgenual ACC remediated this "negativity bias," to the point that patients' ratings of in- volvement with negative stimuli were no different than those of healthy controls. A separate DBS study with the same target and patient population also found reduced post-operative beta oscillatory activity in response to emotionally salient, unpleasant stimuli [66]. Once again, these results further our understanding of how DBS treats refractory psychiatric disorders, and suggest that increased alpha activity in particular brain regions may be a biomarker of MDD.

A different study examining intraoperative activity of the subthalamic nucleus (STN) and local field potentials several days after DBS surgery for OCD found direct neurophysiological evidence of STN involvement in executive function [67]. Specifically, certain populations of STN neurons, distinct from those involved in motor functions, were involved in action inhibition and error monitoring - two executive functions known to be abnormal in OCD [68-71]. Furthermore, DBS of the NAc for OCD results in reduced low-frequency frontal oscillations that are typically evoked by symptom-provoking stimuli, and that are generally associated with the severity of obsessions and compulsions [72].

Finally, in the only study of its kind, Maling et al. [73] (2012) demonstrated increased gamma band activity in the centromedian nucleus of the thalamus to be associated with symptom relief following DBS for Tourette syndrome.

\section{Ablative Surgery}

Compared to DBS, very few attempts have been made to use EEG to predict patients' response to ablative psychiatric surgery. In one of the only studies on this, Evans et al. [74] demonstrated that specific post- 
operative EEG patterns were variably associated with response to subcaudate tractotomy. Specifically, marked high-voltage slow waves with bifrontal localization were most predictive of patient improvement, whereas five other post-operative patterns, ranging from no slow waves to poorly localized slow waves, were less indicative of a successful operation. Although many studies from the mid-to-late 20th century have reported increased slow wave activity as a result of ablative psychiatric surgery, almost none have systematically studied their prognostic utility.

In summary, EEG may offer promising biomarkers of treatment response, which is especially important in surgical treatments where patients are exposed to considerable risks. Having objective predictors of treatment response would be invaluable in selecting good candidates and optimizing the selection of surgical targets. However, research on this topic is predominantly observational, unsystematic, and disjointed. The discovery and validation of reliable EEG biomarkers would require large, systematic, controlled investigations, which are a particular challenge in psychiatric surgery.

\section{Limitations}

Many of the EEG psychiatric surgery studies included in this synthesis are from the mid-20th century, and are confounded by technical limitations, non-standardized EEG acquisition and analysis, heterogeneous criteria for psychiatric diagnoses (i.e., prior to the operationalized criteria of the DSM-III), and small sample sizes. An important consequence of these limitations is that it is unclear whether the post-operative EEG changes represent meaningful functional changes due to psychiatric surgery or are simply a reflection of injury caused by surgical manipulation. Fortunately, less invasive approaches to psychiatric surgery may overcome this problem. For example, MRI-guided focused ultrasound uses ultrasound waves to thermally ablate pathologic brain tissue with sub-millimetre accuracy [3]. These advances in technology allow non-invasive approaches to the brain and would, in theory, minimize any EEG noise caused by neuronal injury. Neurophysiological investigations using these techniques could therefore dramatically improve our understanding of the EEG correlates - if any - of psychiatric neurosurgery.

Despite promising preliminary results for the aforementioned minimally invasive psychosurgical techniques in treating refractory mental illnesses $[75,76]$, there is still a definite need for non-invasive biomarkers to guide the procedures. Furthermore, EEG may help optimize target selection and reveal new targets for circuit disorders. This includes markers that predict treatment response, as well as those that can provide real-time feedback to guide surgeries. The simplicity, portability, and availability of EEG make it an ideal choice for this purpose; however, its application in this domain remains investigational. Our synthesis suggests that there are indeed consistent patterns that can predict the success of psychiatric therapies; however, the lack of large-scale, controlled investigations and validation studies precludes generalizable conclusions.

\section{Conclusion}

In recent years, psychiatric surgery has re-emerged as a viable treatment for debilitating, treatment-refractory mental illnesses. This review summarized the literature on EEG use in the surgical treatment of psychiatric disorders and identified several important themes. First, the EEG correlates of psychiatric neurosurgery are variable, and it is currently unclear whether they are a result of functional changes due to the procedure or simply a reflection of brain injury due to surgical manipulation. Second, intraoperative EEG has been successfully used to guide psychiatric surgery and improve outcomes; however, it requires invasive recording of deep brain structures that is not possible with modern, minimally invasive neurosurgical approaches. Finally, there is considerable evidence supporting the use of EEG as a biomarker for various surgical and non-surgical psychiatric therapies, although large-scale investigations are lacking.

There is a definite need for objective, functional feedback to further improve the safety and accuracy of contemporary psychiatric surgery. EEG offers a promising technique for achieving this, providing real-time neurophysiological correlates to guide procedures. Further systematic study is required to assess its potential as a biomarker for guiding operations and prognosticating outcomes. If successful, the resulting "closed-loop" procedures, combining minimally invasive techniques with functional and structural neurofeedback, have the potential to revolutionize the safety and efficacy of psychiatric surgery. 


\section{Disclosure Statement}

The authors certify that they have no affiliations with or involvement in any organization or entity with any financial interest (such as honoraria; educational grants; participation in speakers' bureaus; membership, employment, consultancies, stock ownership, or other equity interest; and expert testimony or patent-licensing arrangements) or non-financial interest (such as personal or professional relationships, affiliations, knowledge or beliefs) in the subject matter or materials discussed in this paper.

\section{Funding Sources}

No funding was received for this article.

\section{References}

1 Fogwe DT, Mesfin FB. Neuromodulation surgery for psychiatric disorders. StatPearls Publishing; 2018.

2 Lapidus KAB, Kopell BH, Ben-Haim S, Rezai AR, Goodman WK: History of psychosurgery: a psychiatrist's perspective. World Neurosurg. 2013 Sep-Oct;80:S27.e1-16.

3 Meng Y, Suppiah S, Mithani K, Solomon B, Schwartz ML, Lipsman N. Current and emerging brain applications of MR-guided focused ultrasound. J Ther Ultrasound. 2017 Oct;5(1):26.

4 Lipsman N, Mainprize TG, Schwartz ML, Hynynen K, Lozano AM. Intracranial applications of magnetic resonance-guided focused ultrasound. Neurotherapeutics. 2014 Jul;11(3):593-605.

5 National Library of Medicine. Automatic Term Mapping. PubMed Tutor 2018 [cited 2018 Aug 18]. Available from: https://www. nlm.nih.gov/bsd/disted/pubmedtutorial/020_040.html.

6 Cushing/Whitney Medical Library. Yale MeSH Analyzer. Yale University; 2018 [cited 2018 Aug 18]. Available from: http://mesh. med.yale.edu/.

7 Lilian Hutton E, Fleming GW, Fox FE. Early results of prefrontal leucotomy. Lancet. 1941;238(6149):3-7.

8 Davis PA. Electroencephalographic studies on three cases of frontal lobotomy. Psychosom Med. 1941;3(1):38-50.

9 Cohn R. Electroencephalographic study of prefrontal lobotomy. Arch Neurol Psychiatry. 1945;53(4):283.

10 Levin S, Greenblatt M, Healey MM, Solomon HC. Electroencephalographic effects of bilateral prefrontal lobotomy; comparison of cases with and without postlobotomy seizures. Am J Psychiatry. 1949 Sep;106(3): 174-84.

11 Grevilie GD, Last SL: Discussion: Leucotomy as an Instrument of Research. Proc R Soc Med. 1947 Feb;40(4):141-50.

12 Lennox MA, Coolidge J. Electroencephalographic changes after prefrontal lobotomy; with particular reference to the effect of lobotomy on sleep spindles. Arch Neurol Psychiatry. 1949 Aug;62(2):150-61.

13 Stevens H, Mosovich A. Clinical and EEG investigation of prefrontal lobotomy patients. Am J Psychiatry. 1947 Aug;104(2):73-80.
14 Zanello M, Pallud J, Baup N, Peeters S, Turak B, Krebs MO, et al. History of psychosurgery at Sainte-Anne Hospital, Paris, France, through translational interactions between psychiatrists and neurosurgeons. Neurosurg Focus. 2017 Sep;43(3):E9.

15 Bourdillon P, Apra C, Lévêque M, Vinckier F. Neuroplasticity and the brain connectome: what can Jean Talairach's reflections bring to modern psychosurgery? Neurosurg Focus. 2017 Sep;43(3):E11.

16 Henry CE. Effect on the electroencephalogram of transorbital lobotomy. Electroencephalogr Clin Neurophysiol. 1950 May; 2(2):187-92.

17 Holland R, Kopel D, Carmel PW, Prestigiacomo CJ. Topectomy versus leukotomy: J. Lawrence Pool's contribution to psychosurgery. Neurosurg Focus. 2017 Sep;43(3):E7.

18 Krueger EG, Loewenberg Wayne H. Clinical and electroencephalographic effects of prefrontal lobotomy and topectomy in chronic psychoses. AMA Arch Neurol Psychiatry. 1952 May;67(5):661-70.

19 Klotz M. Incidence of seizures, with EEG findings, in prefrontal lobotomy. AMA Arch Neurol Psychiatry. 1955 Aug;74(2):144-8.

20 Le Beau J. Anterior cingulectomy in man. J Neurosurg. 1954 May;11(3):268-76.

21 Walter RD, Yeager CL, Margolis LH, Simon A. The EEG changes in unilateral and bilateral frontal lobotomy. Am J Psychiatry. 1955 Feb;111(8):590-4.

22 Adams CL, Gibbs EL, Gibbs FA. Asynchronism of electrical activity of frontal lobes during sleep; a late sequel of frontal lobotomy. AMA Arch Neurol Psychiatry. 1957 Mar;77(3):237-42.

23 Hosokawa K, Sawada J, Ohara Y, Matsuda K. Follow-up studies on the sleep EEG after prefrontal lobotomy. Polygraphic investigations during spontaneous nocturnal sleep. Folia Psychiatr Neurol Jpn. 1968;22(3):23343.

24 Wildman RW 2nd, Wildman RW. A comparison of the frequency components of the EEGs of lobotomized and control ss: a quantitative approach. J Clin Psychol. 1975 Apr; 31(2):263-6.
25 Benson DF, Stuss DT, Naeser MA, Weir WS, Kaplan EF, Levine HL. The long-term effects of prefrontal leukotomy. Arch Neurol. 1981 Mar;38(3):165-9.

26 Bingley T, Persson A. EEG studies on patients with chronic obsessive-compulsive neurosis before and after psychosurgery (stereotaxic bilateral anterior capsulotomy). Electroencephalogr Clin Neurophysiol. 1978 Jun;44(6):691-6.

27 Mazzini L. Clinical applications of event-related potentials in brain injury. Phys Med Rehabil Clin N Am. 2004 Feb;15(1):163-75.

28 Gomes J, Damborská A. Event-related potentials as biomarkers of mild traumatic brain injury. Act Nerv Super (Praha). 2017; 59(3-4):87-90.

29 Walter WG, Cooper R, Aldridge VJ, McCallum WC, Winter AL. Contingent negative variation: an electric sign of sensorimotor association and expectancy in the human brain. Nature. 1964 Jul;203(4943):380-4

30 Kappenman ES, Luck SJ, editors. The Oxford handbook of event-related potential components. Oxford: Oxford University Press; 2011. https://doi.org/10.1093/oxford$\mathrm{hb} / 9780195374148.001 .0001$.

31 Zappoli R, Denoth F, Papini M, Rossi L, Pasquinelli A, Martinetti MG, et al. [Studies of the expectancy wave (CNV) in patients with interruption of the thalamo-prefrontal interconnnection pathways caused by psychosurgical prefrontal lobotomy operations]. Riv Patol Nerv Ment. 1978 Sep;98(4):233-58.

32 Tecce JJ. A CNV rebound effect. Electroencephalogr Clin Neurophysiol. 1979 May; 46(5):546-51.

33 Tecce JJ, Orzack MH, Mirsky AF. Psychosurgery outcome and CNV asymmetry. Prog Brain Res. 1980;54:709-7.

34 Kuhn J, Gründler TO, Bauer R, Huff W, Fischer AG, Lenartz D, et al. Successful deep brain stimulation of the nucleus accumbens in severe alcohol dependence is associated with changed performance monitoring. Addict Biol. 2011 Oct;16(4):620-3.

35 De Ridder D, Manning P, Glue P, Cape G, Langguth B, Vanneste S. Anterior Cingulate Implant for Alcohol Dependence: case Report. Neurosurgery. 2016 Jun;78(6):E88393. 
36 Beste C, Mückschel M, Elben S, J Hartmann C, McIntyre CC, Saft C, et al. Behavioral and neurophysiological evidence for the enhancement of cognitive control under dorsal pallidal deep brain stimulation in Huntington's disease. Brain Struct Funct. 2015 Jul; 220(4):2441-8.

37 Petersen MC, Dodge HW Jr, Sem-Jacobsen CW, Lazarte JA, Holman CB. Clinical results of selective leukotomy based on intracerebral electrography. J Am Med Assoc. 1955 Oct;159(8):775-6.

38 Holtzheimer PE, Mayberg HS. Deep brain stimulation for psychiatric disorders. Annu Rev Neurosci. 2011;34(1):289-307.

39 Jiménez F, Velasco F, Salin-Pascual R, Hernández JA, Velasco M, Criales JL, et al. A patient with a resistant major depression disorder treated with deep brain stimulation in the inferior thalamic peduncle. Neurosurgery. 2005 Sep;57(3):585-93.

40 Valencia-Alfonso CE, Luigjes J, Smolders R, Cohen MX, Levar N, Mazaheri A, et al. Effective deep brain stimulation in heroin addiction: a case report with complementary intracranial electroencephalogram. Biol Psychiatry. 2012 Apr;71(8):e35-7.

41 Guehl D, Benazzouz A, Aouizerate B, Cuny E, Rotgé JY, Rougier A, et al. Neuronal correlates of obsessions in the caudate nucleus. Biol Psychiatry. 2008 Mar;63(6):557-62.

42 Cohen MX, Axmacher N, Lenartz D, Elger CE, Sturm V, Schlaepfer TE. Nuclei accumbens phase synchrony predicts decisionmaking reversals following negative feedback. J Neurosci. 2009 Jun;29(23):7591-8.

43 Rosa M, Franzini A, Giannicola G, Messina G, Altamura AC, Priori A. Hypothalamic oscillations in human pathological aggressiveness. Biol Psychiatry. 2012 Dec;72(12):e335.

44 Smolders R, Mazaheri A, van Wingen G, Figee M, de Koning PP, Denys D. Deep brain stimulation targeted at the nucleus accumbens decreases the potential for pathologic network communication. Biol Psychiatry. 2013 Nov;74(10):e27-8.

45 McIntyre RS, Filteau MJ, Martin L, Patry S, Carvalho A, Cha DS, et al. Treatment-resistant depression: definitions, review of the evidence, and algorithmic approach. J Affect Disord. 2014 Mar;156:1-7.

46 Olbrich S, Arns M. EEG biomarkers in major depressive disorder: discriminative power and prediction of treatment response. Int Rev Psychiatry. 2013 Oct;25(5):604-18.

47 Baskaran A, Milev R, Mcintyre RS: The neurobiology of the EEG biomarker as a predictor of treatment response in depression. Neuropharmacology. 2012 Sep;63(4):50713.

48 Pizzagalli DA. Frontocingulate dysfunction in depression: toward biomarkers of treatment response. Neuropsychopharmacology. 2011 Jan;36(1):183-206.
49 van der Vinne N, Vollebregt MA, van Putten MJ, Arns M. Frontal alpha asymmetry as a diagnostic marker in depression: fact or fiction? A meta-analysis. Neuroimage Clin. 2017 Jul;16:79-87.

50 Fidalgo TM, Morales-Quezada JL, Muzy GS, Chiavetta NM, Mendonca ME, Santana MV, et al. Biological markers in noninvasive brain stimulation trials in major depressive disorder: a systematic review. J ECT. 2014 Mar; 30(1):47-61.

51 Olbrich S, van Dinteren R, Arns M. Personalized Medicine: Review and Perspectives of Promising Baseline EEG Biomarkers in Major Depressive Disorder and Attention Deficit Hyperactivity Disorder. Neuropsychobiology. 2015;72(3-4):229-40.

52 Wade EC, Iosifescu DV. Using Electroencephalography for Treatment Guidance in Major Depressive Disorder. Biol Psychiatry Cogn Neurosci Neuroimaging. 2016 Sep; 1(5):411-22.

53 Kobayashi B, Cook IA, Hunter AM, Minzenberg MJ, Krantz DE, Leuchter AF. Can neurophysiologic measures serve as biomarkers for the efficacy of repetitive transcranial magnetic stimulation treatment of major depressive disorder? Int Rev Psychiatry. 2017 Apr;29(2):98-114.

54 Frodl T. Recent advances in predicting responses to antidepressant treatment. F1000 Res. 2017 May;6:F1000 Faculty Rev-619.

55 Baskaran A, Farzan F, Milev R, Brenner CA, Alturi S, Pat McAndrews M, et al.; CANBIND Investigators Team. The comparative effectiveness of electroencephalographic indices in predicting response to escitalopram therapy in depression: A pilot study. J Affect Disord. 2018 Feb;227:542-9.

56 Pascual-Marqui RD, Michel CM, Lehmann D. Low resolution electromagnetic tomography: a new method for localizing electrical activity in the brain. Int J Psychophysiol. 1994 Oct;18(1):49-65.

57 Pascual-Marqui RD, Esslen M, Kochi K, Lehmann D. Functional imaging with lowresolution brain electromagnetic tomography (LORETA): a review. Methods Find Exp Clin Pharmacol. 2002;24 Suppl C:91-5.

58 Khodayari-Rostamabad A, Reilly JP, Hasey GM, de Bruin H, Maccrimmon DJ. A machine learning approach using EEG data to predict response to SSRI treatment for major depressive disorder. Clin Neurophysiol. 2013 Oct;124(10):1975-85.

59 Erguzel TT, Ozekes S, Gultekin S, Tarhan N, Hizli Sayar G, Bayram A. Neural Network Based Response Prediction of rTMS in Major Depressive Disorder Using QEEG Cordance. Psychiatry Investig. 2015 Jan;12(1): 61-5.

60 Quraan MA, Protzner AB, Daskalakis ZJ, Giacobbe $\mathrm{P}$, Tang CW, Kennedy $\mathrm{SH}$, et al. EEG power asymmetry and functional connectivity as a marker of treatment effectiveness in DBS surgery for depression. Neuropsychopharmacology. 2014 Apr;39(5):1270-81.
61 Smart OL, Tiruvadi VR, Mayberg HS. Multimodal approaches to define network oscillations in depression. Biol Psychiatry. 2015 Jun;77(12):1061-70.

62 Alhourani A, McDowell MM, Randazzo MJ, Wozny TA, Kondylis ED, Lipski WJ, et al. Network effects of deep brain stimulation. J Neurophysiol. 2015 Oct;114(4):2105-17.

63 Neumann WJ, Huebl J, Brücke C, Gabriëls L, Bajbouj M, Merkl A, et al. Different patterns of local field potentials from limbic DBS targets in patients with major depressive and obsessive compulsive disorder. Mol Psychiatry. 2014 Nov; 19(11):1186-92.

64 Clark DL, Brown EC, Ramasubbu R, Kiss $\mathrm{ZH}$. Intrinsic Local Beta Oscillations in the Subgenual Cingulate Relate to Depressive Symptoms in Treatment-Resistant Depression. Biol Psychiatry. 2016 Dec;80(11):e934.

65 Merkl A, Neumann WJ, Huebl J, Aust S, Horn A, Krauss JK, et al. Modulation of BetaBand Activity in the Subgenual Anterior Cingulate Cortex during Emotional Empathy in Treatment-Resistant Depression. Cereb Cortex. 2016 Jun;26(6):2626-38

66 Huebl J, Brücke C, Merkl A, Bajbouj M, Schneider GH, Kühn AA. Processing of emotional stimuli is reflected by modulations of beta band activity in the subgenual anterior cingulate cortex in patients with treatment resistant depression. Soc Cogn Affect Neurosci. 2016 Aug;11(8):1290-8.

67 Bastin J, Polosan M, Benis D, Goetz L, Bhattacharjee M, Piallat B, et al. Inhibitory control and error monitoring by human subthalamic neurons. Transl Psychiatry. 2014 Sep; 4(9):e439.

68 Roh D, Chang JG, Yoo SW, Shin J, Kim CH. Modulation of error monitoring in obsessive-compulsive disorder by individually tailored symptom provocation. Psychol Med. 2017 Sep;47(12):2071-80.

69 Berlin GS, Lee HJ. Response inhibition and error-monitoring processes in individuals with obsessive-compulsive disorder. J Obsessive Compuls Relat Disord. 2018 Jan;16: 21-7.

70 Lei H, Zhu X, Fan J, Dong J, Zhou C, Zhang $\mathrm{X}$, et al. Is impaired response inhibition independent of symptom dimensions in obsessive-compulsive disorder? Evidence from ERPs. Sci Rep. 2015 May;5(1):10413.

71 Morein-Zamir S, Fineberg NA, Robbins TW, Sahakian BJ. Inhibition of thoughts and actions in obsessive-compulsive disorder: extending the endophenotype? Psychol Med. 2010 Feb;40(2):263-72.

72 Figee M, Luigjes J, Smolders R, Valencia-Alfonso CE, van Wingen G, de Kwaasteniet B, et al. Deep brain stimulation restores frontostriatal network activity in obsessive-compulsive disorder. Nat Neurosci. 2013 Apr; 16(4):386-7. 
73 Maling N, Hashemiyoon R, Foote KD, Okun MS, Sanchez JC. Increased thalamic gamma band activity correlates with symptom relief following deep brain stimulation in humans with Tourette's syndrome. PLoS One. 2012; 7(9):e44215.

74 Evans BM, Bridges PK, Bartlett JR. Electroencephalographic changes as prognostic indicators after psychosurgery. J Neurol Neurosurg Psychiatry. 1981 May;44(5):444-7.
75 Kim SJ, Roh D, Jung HH, Chang WS, Kim $\mathrm{CH}$, Chang JW. A study of novel bilateral thermal capsulotomy with focused ultrasound for treatment-refractory obsessivecompulsive disorder: 2-year follow-up. J Psychiatry Neurosci. 2018 May; 43(4): 170188.

76 Miguel EC, Lopes AC, McLaughlin NC, Norén G, Gentil AF, Hamani C, et al. Evolution of gamma knife capsulotomy for intractable obsessive-compulsive disorder. Mol Psychiatry. 2019 Feb;24(2):218-240.
77 Moher D, Liberati A, Tetzlaff J, Altman DG; PRISMA Group. Preferred reporting items for systematic reviews and meta-analyses: the PRISMA statement. PLoS Med. 2009 Jul; 6(7):e1000097.

78 Hosokawa K, Kawada Y, Matsuda K, Usui S, Hikiji A, Ninomiya K. EEG studies on 100 cases of frontal lobotomy. Proceedings of the 15th Annual Meeting of Japan EEG Society; 1966. p. 57-59. 